Оригинальная статья/Original article

УДК 519.712

DOI: http://doi.org/10.20914/2310-1202-2016-3-135-143

\begin{tabular}{c} 
Математическое моделирование влияния внешних воздействий \\
на температурные показатели масел двигателя внутреннего \\
сгорания и элементов трансмиссии \\
\hline
\end{tabular}

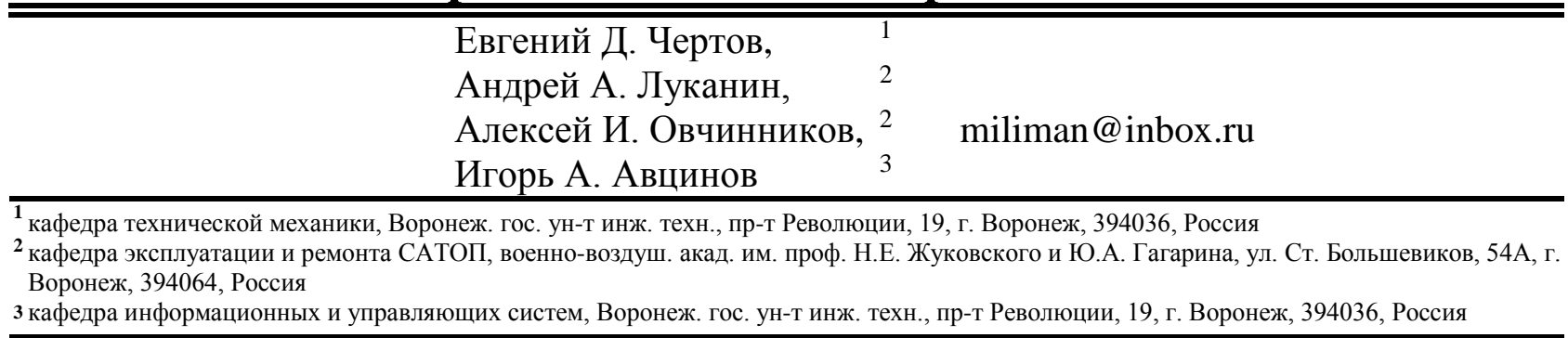

Реферат. Для описания процессов, происходящих в двигателе внутреннего сгорания и элементах трансмиссии, используются различные математические модели. В работе применяется раздел математической статистики - планирование эксперимента. Для оценки влияния внешних воздействий на температурные показатели масел ДВС и элементов трансмиссии был реализован полный факторный эксперимент, который направлен на определение температуры масла ДВС, коробки передач и главных передач. Моделирование влияния внешних воздействий на температурные показатели масел ДВС и элементов трансмиссии заключалось в следующем: строилась концептуальная модель; проводилась её формализация и исследование моделируемого процесса; определялись необходимые аппроксимации; строилась обобщённая схема процесса; по этой обобщённой схеме создавался алгоритм и разрабатывался программный комплекс; проводились расчёты на ЭВМ; определялись математические и графические зависимости внешних воздействий на температурные показатели масел ДВС и элементов трансмиссии; разрабатывались практические рекомендации по процессу подогрева применительно к эксплуатационным условиям СНО ОП. Объектом исследования является температура окружающего воздуха, время работы системы подогрева и мощность теплового агрегата, влияющие на температуру масел ДВС, коробки передач, главных передач ВАТ. Задача сводится к определению такой мощности теплового агрегата, при которой достигается максимальное значение температуры масла за минимальное время. Спланирован полный факторный эксперимент ПФЭ позволяющий определять взаимодействие двух факторов, которое и проявляется при одновременном варьировании этих факторов при действие каждого из них на выход в зависимости от уровня их нахождения.

Ключевые слова: математическое моделирование, температурные показатели, ДВС, элементы трансмиссии

\title{
Mathematical modeling of the impact of external influences on the temperature behavior of the internal combustion engine oils and transmission components
}

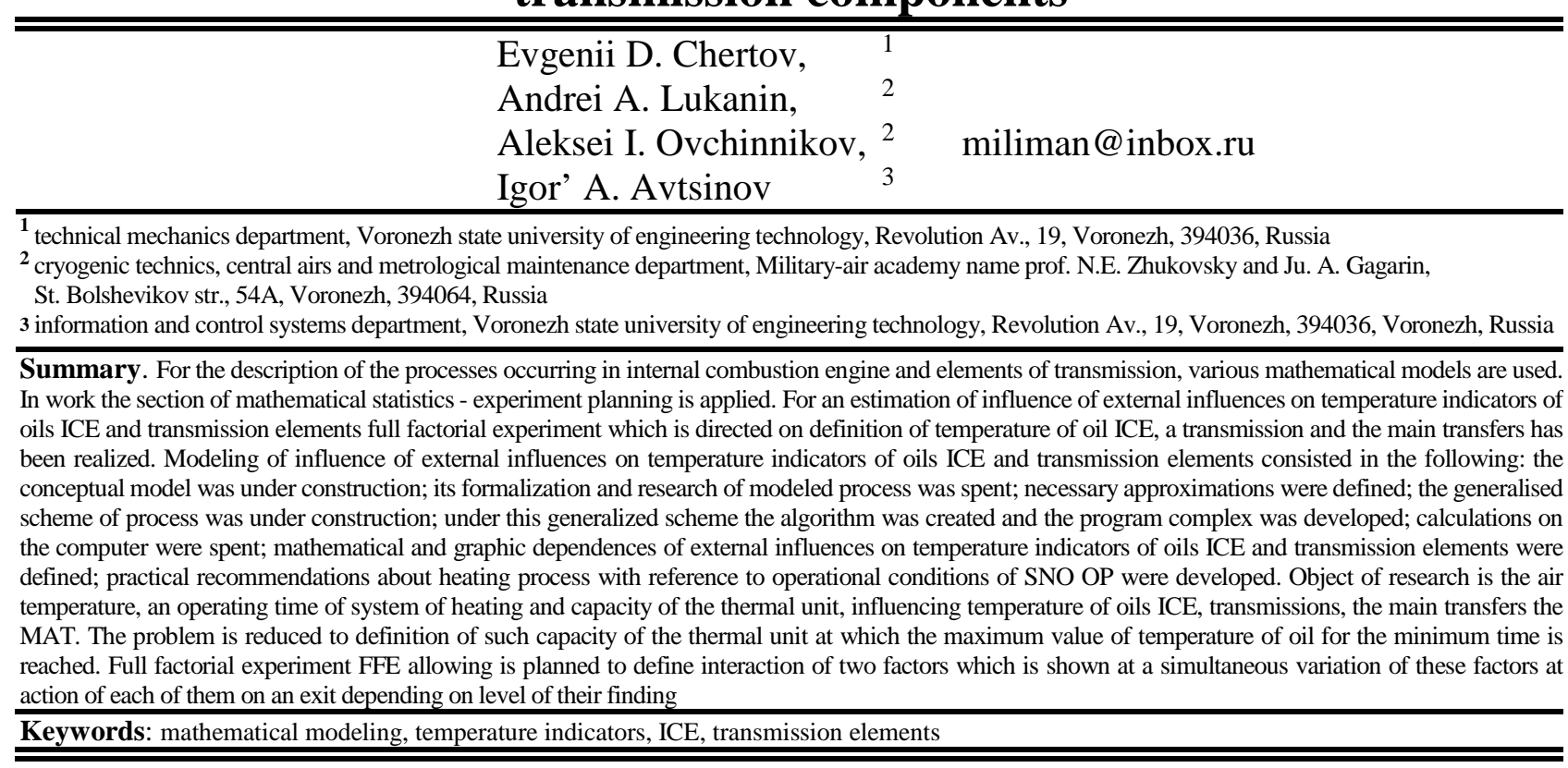

Для цитирования

Чертов Е. Д., Луканин А. А., Овчинников А. И., Авцинов И. А. Математическое моделирование влияния внешних воздействий на температурные показатели масел двигателя внутреннего сгорания и элементов трансмиссии // Вестник ВГУИТ. 2016. № 3. С. 135-143. doi:10.20914/2310-1202-2016-3-135-143
For citation

Chertov E. D., Lukanin A. A., Ovchinnikov A. I., Avtsinov I. A. Mathematical modeling of the impact of external influences on the temperature behavior of the internal combustion engine oils and transmission components. Vestnik VSUET [Proceedings of VSUET]. 2016. no. 3. pp. 135-143. (in Russian). doi:10.20914/2310-1202-2016-3-135-143 


\section{Введение}

Проведенный анализ работ $[2,4,5]$ показывает, что для описания процессов, происходящих в ДВС и элементах трансмиссии, используются различные математические модели. В данной работе применяется раздел математической статистики - планирование эксперимента. Для оценки влияния внешних воздействий на температурные показатели масел ДВС и элементов трансмиссии был реализован полный факторный эксперимент, который направлен на определение температуры масла ДВС, коробки передач и главных передач. Моделирование влияния внешних воздействий на температурные показатели масел ДВС и элементов трансмиссии заключалось в следующем: строилась концептуальная модель; проводилась её формализация и исследование моделируемого процесса; определялись необходимые аппроксимации; строилась обобщённая схема процесса; по этой обобщённой схеме создавался алгоритм и разрабатывался программный комплекс; проводились расчёты на ЭВМ; определялись математические и графические зависимости внешних воздействий на температурные показатели масел ДВС и элементов трансмиссии; разрабатывались практические рекомендации по процессу подогрева применительно к эксплуатационным условиям СНО ОП.

Подогрев масел двигателя и элементов трансмиссии осуществляется системой подогрева, принципиальная схема которой представлена на рисунке 1.

Объектом исследования на данном этапе исследований (полного факторного эксперимента) является температура окружающего воздуха, время работы системы подогрева и мощность теплового агрегата, влияющие на температуру масел ДВС, коробки передач, главных передач ВАТ.

Задача сводится к определению такой мощности теплового агрегата, при которой достигается максимальное значение температуры масла за минимальное время. Факторы, влияющие температуру масла ДВС, КП, ГП, представлены на рисунке 2.

Выбираются основные входные (управляемые) факторы, определяющие условия работы системы подогрева: $T_{\text {ов }}$ - температура окружающей среды, ${ }^{\circ} \mathrm{C} ; t-$ время работы системы подогрева, мин; $W_{T A}-$ мощность теплового агрегата, кВт.

За основные оценочные показатели (выходные факторы) принимаются показатели температуры масла: температура масла ДВС $T_{\text {двС }},{ }^{\circ} \mathrm{C}$; температура масла КП $T_{\kappa \Pi,}{ }^{\circ} \mathrm{C}$; температура масла ГП $T_{\Gamma \Pi,}{ }^{\circ} \mathrm{C}$.

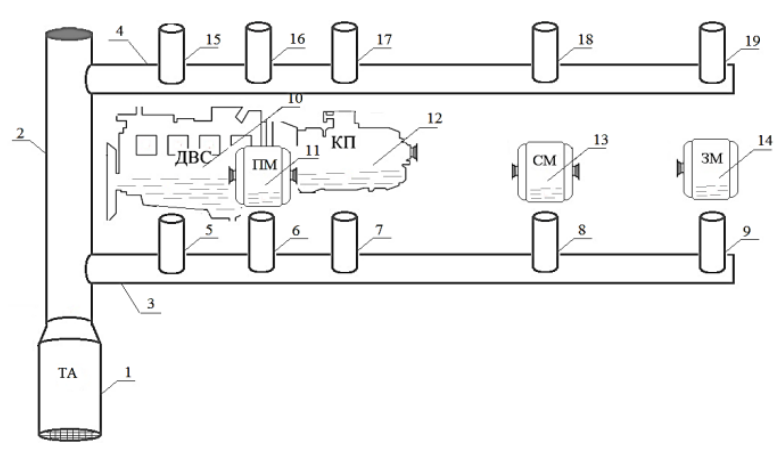

Рисунок 1. Принципиальная схема системы подогрева масел двигателя и элементов трансмиссии: 1 - тепловой агрегат; 2- основная магистраль подвода теплоты; 3,4 - воздуховод подвода теплоты к стоянке автомобильной техники; 5,15 - воздуховод подвода теплоты к картеру масла ДВС; 6,16 - воздуховод подвода теплоты к картеру масла переднего моста ПМ; 7,17 - воздуховод подвода теплоты к картеру масла коробки передач КП; 8,18 - воздуховод подвода теплоты к картеру масла среднего моста СМ; 9,19 - воздуховод подвода теплоты к картеру масла заднего моста 3М; 10 - двигатель внутреннего сгорания; 11 - передний мост; 12 - коробка передач; 13 - средний мост; 14 - задний мост.

Figure 1. The concept of heating engine oils and transmission elements: 1 - heat unit; 2 - the main artery supplying heat; 3,4 - duct heat supply to the Parking of automotive vehicles; 5,15 - duct heat supply to the crankcase oil of the internal combustion engine; 6,16-duct heat supply to the crankcase oil of the front axle PM; 7,17 - duct heat supply to the crankcase oil transmission KP; for 8, 18 - air duct for supplying heat to the crankcase oil of the middle bridge CM; 9,19-duct heat supply to the crankcase oil of the rear axle ZM; 10 - an internal combustion engine; 11 - rear axle; 12 - transmission; 13 - median bridge; 14 - rear axle.

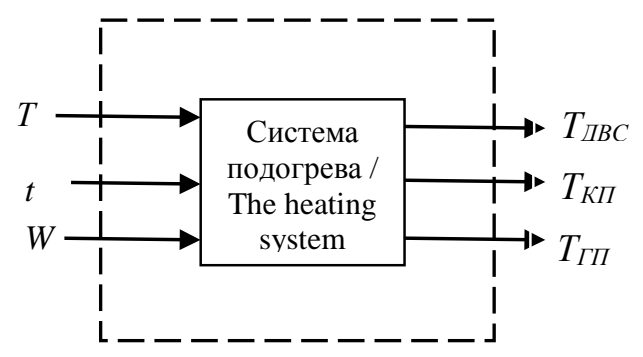

Рисунок 2. Факторы, влияющие на эксплуатационные и экологические показатели работы ДВС

Figure 2. . Factors affecting the operational and environmental indicators of work ICE

В основу планирования эксперимента был заложен план полного факторного эксперимента (ПФЭ), в котором исследуемые факторы изменялись на двух уровнях: нижнем $x_{i н}$ и верхнем $x_{i 6}$, симметрично расположенных относительно основного уровня $x_{i 0}$. [3]. Изображение плана ПФЭ при кодовом обозначении значений величин факторов показано на рисунке 3. 


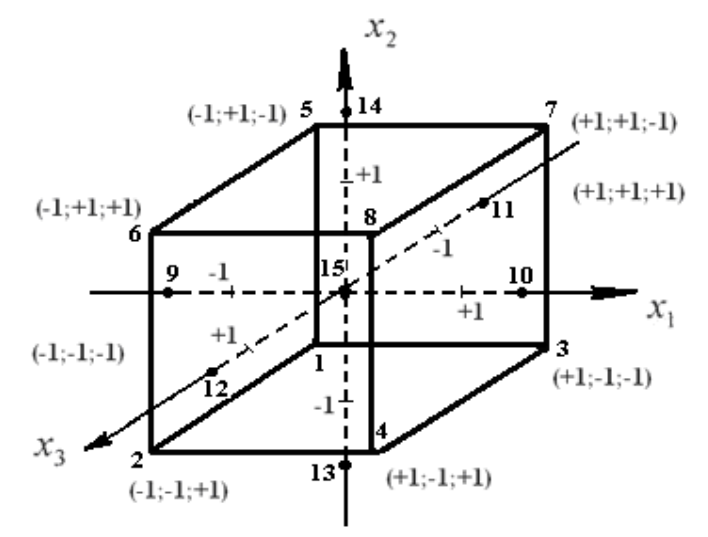

Рисунок 3. Графическое изображение ПФЭ

Figure 3. Graphic image of PPE

Построению плана ПФЭ предшествовало проведение ряда неформализованных действий (принятие решений), направленных на выбор локальной области факторного пространства, исходя из свойств исследуемого объекта.

Общее число опытов в плане для ПФЭ было равно:

$$
\mathrm{N}=\mathrm{NI}+\mathrm{NII}+\mathrm{NIII},
$$

где $\mathrm{NI}=2 \mathrm{R}=8-$ число всех возможных наблюдений; $\mathrm{NII}=2 \mathrm{R}=6$, где $\mathrm{R}-$ количество факторов; $\mathrm{NIII}=6$; тогда $\mathrm{N}=20$, а плечо $\mathrm{r}=2 \mathrm{R} / 4=1,682$.

Вначале выбираются основные (нулевые) уровни хі́0, затем - интервалы варьирования $\Delta x i ́$ и определяются нижние хі́н и верхние хі́в уровни. В качестве исходной точки хі́0 выбирали такую, которая соответствовала наилучшим условиям, определенным на основе анализа априорной информации о системе. На выбор интервала варьирования $\Delta x i ́$ накладывались естественные ограничения снизу (интервал не мог быть меньше ошибки фиксирования уровня фактора, так как в противном случае верхний и нижний уровни оказались бы неразличимыми) и сверху (верхний и нижний уровни не должны выходить за область определения).

Предельные значения входных (управляемых) факторов (таблица 1) устанавливаются в результате проведения предварительных опытов и анализа, выполненных ранее работ.

Уровни выходных факторов и интервалы варьирования отображены в таблице 2. Выбирая комбинации уровней факторов для каждой экспериментальной точки квадрата, получается план ПФЭ (таблица 3).

Таблица 1

Предельные значения входных (управляемых) факторов

Table 1

The limit values of the input (driven) factors

\begin{tabular}{|c|c|c|c|c|c|c|c|c|}
\hline \multirow{2}{*}{$\begin{array}{c}\text { Факторы / } \\
\text { Factors }\end{array}$} & \multirow{2}{*}{$\begin{array}{l}\text { Кодовое обо- } \\
\text { значение } \\
\text { A coded } \\
\text { indication }\end{array}$} & \multirow{2}{*}{$\begin{array}{l}\text { Обозначение } \\
\text { Indication }\end{array}$} & \multirow{2}{*}{$\begin{array}{l}\text { Интервал варь- } \\
\text { ирования } \\
\text { The interval of } \\
\text { variation }\end{array}$} & \multicolumn{5}{|c|}{$\begin{array}{c}\text { Уровни варьирования } \\
\text { Levels of variation }\end{array}$} \\
\hline & & & & $-1,682$ & -1 & 0 & 1 & 1,682 \\
\hline $\begin{array}{l}\text { Температура окружающего } \\
\text { воздуха, }{ }^{\circ} \mathrm{C} / \\
\text { Ambient temperature, }{ }^{\circ} \mathrm{C}\end{array}$ & $x_{1}$ & $T_{\text {ов }}$ & 10 & -36 & -29 & -19 & -9 & -2 \\
\hline $\begin{array}{l}\text { Время работы системы по- } \\
\text { догрева, мин / } \\
\text { Working hours of the heating } \\
\text { system, min }\end{array}$ & $x_{2}$ & $t$ & 10 & 3 & 10 & 20 & 30 & 37 \\
\hline $\begin{array}{l}\text { Мощность теплового агре- } \\
\text { гата, кВт / } \\
\text { Power thermal unit, kW }\end{array}$ & $x_{3}$ & $W_{T . A .}$ & 20 & 12 & 20 & 40 & 60 & 74 \\
\hline
\end{tabular}

Уровни входных факторов и интервалы варьирования

Таблица 2

Table 2

The levels of the input factors and ranges of variation

\begin{tabular}{|c|c|c|}
\hline \multirow{2}{*}{$\begin{array}{c}\text { Bходной (управляемый) фактор / } \\
\text { Input (controlled) factor }\end{array}$} & \multicolumn{2}{|c|}{$\begin{array}{c}\text { 3начение факторов / } \\
\text { Meaning factors }\end{array}$} \\
\cline { 2 - 3 } & Hижнеe / & Bepхнеe / \\
& Lower & $x_{i s}$ \\
\hline$T_{o s},{ }^{\circ} \mathrm{C}$ & -29 & -9 \\
\hline$t$, мин & 10 & 30 \\
\hline$W_{T A}, \mathrm{\kappa BT}$ & 20 & 60 \\
\hline
\end{tabular}


План полного факторного эксперимента ПФЭ

Table 3

Design of full factorial experiment PPE

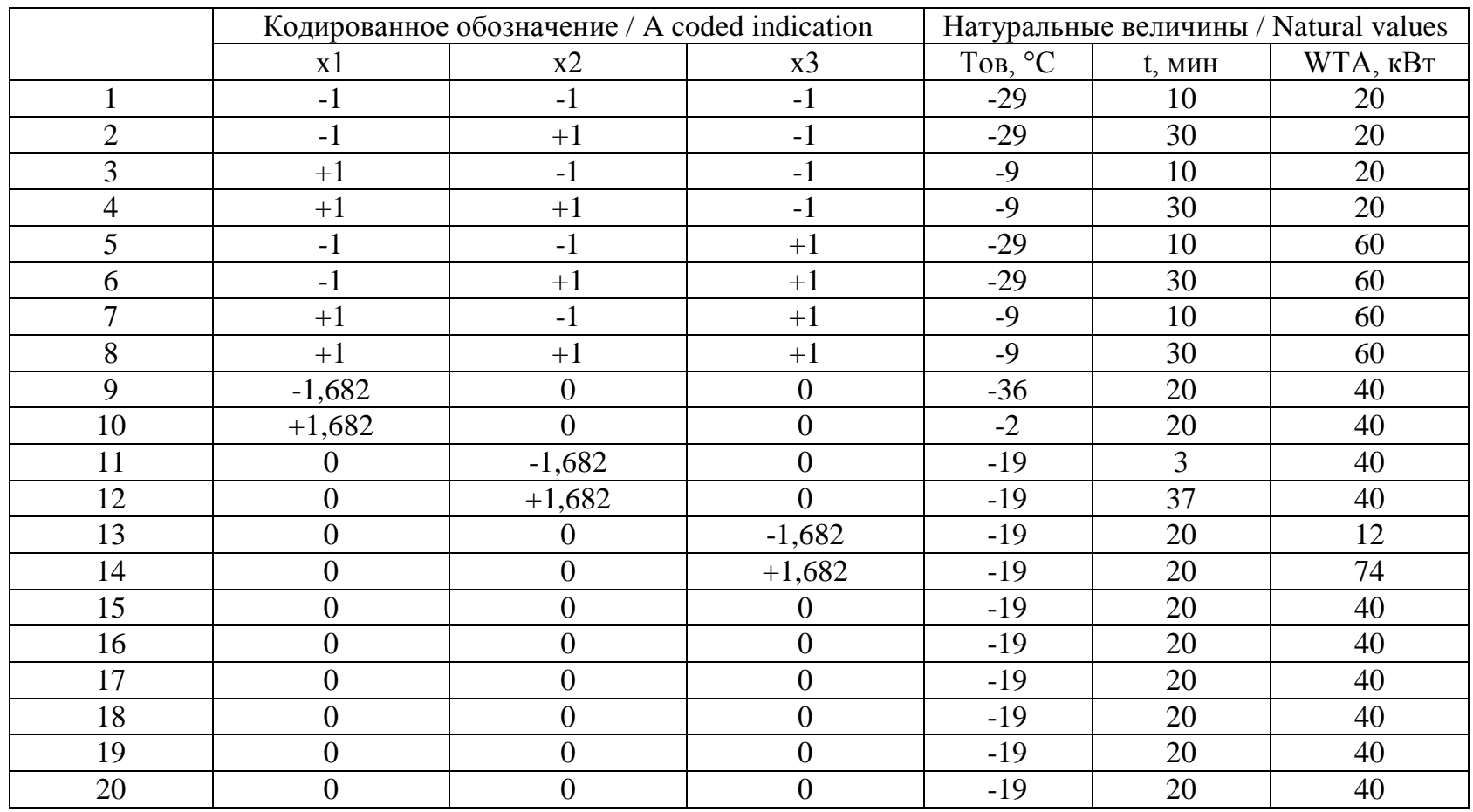

Предполагается влияние трех количественных факторов $(R=3) x_{i}(i=\overline{1, R})$ на некоторую реакцию $y_{u}$ в отведенной для экспериментирования локальной области факторного пространства, предельные значения. Тогда математической моделью влияния внешних воздействий на температурные показатели масел ДВС и элементов трансмиссии будут соотношения вида:

$$
\begin{aligned}
& y_{g e}=f_{M}\left(x_{1}, x_{2}, x_{3}\right) ; \\
& y_{C O}=f_{m}\left(x_{1}, x_{2}, x_{3}\right) ; \\
& y_{C H}=f_{I}\left(x_{1}, x_{2}, x_{3}\right) .
\end{aligned}
$$

Допускается, что каждую из функций (2) можно с допустимой степенью точности представить в виде полинома второй степени от трех переменных, который содержит десять коэффициентов. Тогда каждая из функций $y_{g e}$, $y_{C O}, y_{C H}$ будут иметь вид [3]:

$$
y=b_{o}+\sum_{l=1}^{3} b_{t} x_{t}+\sum_{l \succ \mathrm{j}}^{3} b_{t j} x_{t} \mathrm{x}_{\mathrm{j}}+\sum_{l=1}^{3} b_{l t} x_{l}^{2},
$$

где $b_{o}-$ свободный член уравнения, определяется при условии, что рассматриваемые факторы находятся на средних «нулевых» условиях; $x$ - масштабированные значения факторов, которые определяют функцию и поддаются варьированию; $l, j$ - индексы факторов; $b_{l}$ - коэффициент при линейных членах; $b_{i j}$ - коэффициенты двухфакторных взаимодействий, показывающие как изменяется степень влияния одного фактора при изменении величины другого; $b_{l i}-$ коэффициенты при квадратичных членах.

После выбора модели планирования ПФЭ, изучаемого процесса определяем функции $y=f\left(x_{1}, x_{2} \ldots, x_{R}\right)$ и её уравнения в отведенной для исследования области факторного пространства. При этом необходимо спланировать и провести эксперименты для оценки числовых значений коэффициентов этого уравнения. Основная задача планирования эксперимента получение максимального объема необходимой информации об объекте моделирования, заданном в виде алгоритма [3].

По результатам трёхфакторного эксперимента составляется уравнение регрессии, в котором помимо линейных членов присутствуют члены, учитывающие эффекты парных межфакторных взаимодействий. Уравнение регрессии второго порядка в 3 - факторном эксперименте имеет вид: $\mathrm{y}=\mathrm{b}_{0}+\mathrm{b}_{1} \mathrm{x}_{1}+\mathrm{b}_{2} \mathrm{x}_{2}+\mathrm{b}_{3} \mathrm{X}_{3}+\mathrm{b}_{12} \mathrm{x}_{1} \mathrm{x}_{2}+$ $+b_{13} X_{1} X_{3}+b_{23} X_{2} X_{3}+b_{11} X_{1}^{2}+b_{22} X_{2}^{2}+b_{33} X_{3}^{2}$. (4) 
План ПФЭ $2^{3}$ даёт возможность рассчитать 10 коэффициентов. Коэффициенты при членах уравнения регрессии (4) для ПФЭ $2^{3}$ рассчитываются по выражениям [3]:

$$
\begin{gathered}
b_{0}=a_{0} \sum_{u=1}^{N} y_{u}-a_{4} \sum_{i=1}^{R} \sum_{u=1}^{N-N_{I I}} x_{i u}^{2} y_{u} \\
b_{i}=a_{1} \sum_{u=1}^{N-N_{I I}} x_{i u} y_{u} \\
b_{i j}=a_{2} \sum_{u=1}^{N_{I}} x_{i u} x_{j u} y_{u} ; \\
b_{i i}=\frac{a_{2}}{2} \sum_{u=1}^{N-N_{I I I}} x_{i u}^{2} y_{u}+a_{3} \sum_{i=1}^{R} \sum_{u=1}^{N-N_{I I I}} x_{i u}^{2} y_{u}-a_{4} \sum_{u=1}^{N} y_{u}
\end{gathered}
$$

где $a_{0}=0,16634 ; a_{1}=0,07322 ; a_{2}=0,12500 ; a_{3}=$ 0,$00689 ; a_{4}=0,05679$. При расчете коэффициентов уравнения были использованы средние результаты опытов $(\mathrm{N})$, рассчитанные по достоверным результатам отдельных повторностей. Средняя оценка результата u-го опыта рассчитывалась по формуле:

$$
\bar{y}_{u}=\frac{1}{m_{u}} \sum_{k=1}^{m_{u}} y_{u k},
$$

где $m_{u}$ - число повторностей $u$-го опыта, $m_{u}=$ const $=m=3$.

ПФЭ не является полностью ортогональными. Так как $\sum_{u=1}^{N} x_{i u} x_{j u}=0(i=0 \div R, j=1 \div R, i$ $\neq j)$, то $\sum_{u=1}^{N} x_{0 u} x_{i u}^{2} \neq 0 \quad$ и $\sum_{u=1}^{N} x_{i u}^{2} x_{j u}^{2} \neq 0$. Поэтому по результатам реализации плана ПФЭ оцениваются лишь линейные эффекты факторов $b_{i}$ и эффекты межфакторных взаимодействий $b_{i j}$. Коэффициенты $b_{0}$ и $b_{i i}$ коррелированны между собой, что усложняло процедуру вычислений этих коэффициентов и статистического анализа их значимости. Статистический анализ коэффициентов уравнений регрессии, описывающих процесс подогрева масел двигателя и элементов трансмиссии системой подогрева

Определение выхода процесса и обеспечение заданного уровня факторов в каждом опыте осуществлялись с ошибкой. Следовательно, с этой ошибкой определялись и коэффициенты уравнения регрессии.

Для того, чтобы показать с заранее заданной вероятностью $P$, что полученные оценки коэффициентов уравнения по модулю либо больше (тогда они значимо отличаются от нуля), либо меньше ошибки в их определении (тогда они незначимо отличаются от нуля и должны быть из уравнения исключены) был проведен статистический анализ уравнения.

Определение построчной оценки дисперсии воспроизводимости единичного результата измерения в каждом опыте осуществлялось по формуле:

$$
S^{2}\left(y_{u k}\right)=\frac{\sum_{k=1}^{m}\left(y_{u k}-\bar{y}_{u}\right)^{2}}{m-1} .
$$

Если измеряемая случайная величина $y_{u k}$ распределена по нормальному закону во всем исследованном диапазоне, то независимо от значений $\bar{y}_{u}$ дисперсия $\sigma^{2}$ не будет изменять своей величины. Следовательно, оценки этой дисперсии должны быть однородными (являющимися оценкой одного и того же параметра, в данном случае - дисперсии $\sigma^{2}$ ).

Так как $m_{u}=m=$ const, то однородность оценок дисперсий анализировалась при помощи критерия Кохрена $G$ :

$$
G=\frac{\left[S^{2}\left(y_{u k}\right)\right]_{\max }}{\sum_{u=1}^{N} S^{2}\left(y_{u k}\right)}
$$

где $\left[S^{2}\left(y_{u k}\right)\right]_{\max }$ - максимальная дисперсия; $\sum_{u=1}^{N} S^{2}\left(y_{u k}\right)$ - сумма всех дисперсий.

Далее сравнивалось это отношение с критическим значением критерия Кохрена $G_{k p}$. Если $G<G_{\kappa p}$, то оценки однородны.

Критическое значение критерия Кохрена находилось по таблицам [1] в зависимости от числа степеней свободы числителя $f_{1}$, числа сравниваемых дисперсий $N$ и принятого уровня значимости $q=0,05$.

Средняя для всего эксперимента оценка дисперсии воспроизводимости единичного результата рассчитывалась по формуле:

$$
S^{2}\left(y_{k}\right)=\frac{\sum_{u=1}^{N} S^{2}\left(y_{u k}\right)}{N}=\frac{\sum_{u=1}^{N} \sum_{k=1}^{m}\left(y_{u k}-\bar{y}_{u}\right)^{2}}{N(m-1)} .
$$

Величина $\bar{y}_{u}$ определялась по m повторностям, следовательно, она была ближе к истинному значению выхода, чем результаты единичных повторностей в соответствующем варианте опыта. Средняя для всего 
эксперимента дисперсия воспроизводимости среднего значения выхода в каждой строке была в т раз меньше дисперсии $S^{2}\left(y_{k}\right)$, т. е.:

$$
S^{2}(\bar{y})=\frac{S^{2}\left(y_{k}\right)}{m}=\frac{\sum_{u=1}^{N} \sum_{k=1}^{m}\left(y_{u k}-\bar{y}_{u}\right)^{2}}{N(m-1) m} .
$$

В определении оценки любого из коэффициентов уравнения участвовали все $N$ средних результатов опытов, оценкой дисперсии которых была одна и та же величина $S^{2}(\bar{y})$, т. е. оценка дисперсии любого из независимо определяемых коэффициентов имела одну и ту же величину.

Структура формул для определения оценок коэффициентов была одинаковой со структурой формулы для расчета среднеарифметического:

$$
S^{2}\left(b_{i}\right)=\frac{S^{2}(\bar{y})}{N}=\frac{\sum_{u=1}^{N} \sum_{k=1}^{m}\left(y_{u k}-\bar{y}_{u}\right)^{2}}{N(m-1) m N}
$$

Доверительная ошибка коэффициентов $\varepsilon\left(b_{i}\right)$ рассчитывалась по критерию Стьюдента $t(P ; f)$ :

$$
\varepsilon\left(b_{i}\right)=t(P ; f)\left(S\left(b_{i}\right)\right),
$$

где $\mathrm{S}\left(b_{i}\right)=\sqrt{S^{2}\left(b_{i}\right)}-$ оценка коэффициентов уравнения регрессии.

Полученные коэффициенты регрессии $b_{i}$ сравнивались с доверительной ошибкой $\varepsilon\left(b_{i}\right)$. Если $\left|b_{i}\right|>\varepsilon\left(b_{i}\right)$, то оценка коэффициента $b_{i}$ значимо отличалась от нуля. В противном случае оценка $b_{i}$ считалась значимо, не отличающейся от нуля и приравнивалась нулю.

При получении незначительного линейного коэффициента какого-либо фактора искалось этому объяснение, проанализировав следующие ситуации:

- данный фактор на исследуемый процесс не влияет;

- выбран слишком малый интервал варьирования, в связи, с чем изменение выхода процесса, обусловленное изменением фактора, соразмерно случайным отклонениям, вызываемым влиянием неучтенных факторов или погрешностью измерительных приборов;

- значение данного фактора в центральной точке эксперимента соответствует его оптимальной величине, в связи, с чем одинаковое его уменьшение или увеличение на $\Delta x_{i}$ понизит выход процесса приблизительно на одну и ту же величину.
Если в уравнении после проверки значимости коэффициентов оставались все $N$ коэффициентов, то проверка адекватности уравнения теряла смысл. Рассчитанный по такому уравнению выход процесса для условий какого-либо u-го опыта должен в пределах точности округления совпадать с величиной $\bar{y}_{i}$, принятой к расчету.

Если число значимых коэффициентов хотя бы на единицу было меньше числа опытов, то возникала необходимость (и возможность) статистической проверки адекватности уравнения экспериментальным данным.

Проверка адекватности (соответствия) математической модели экспериментальным данным осуществляется по критерию Фишера - F, которое сравнивалось с табличным значением критерия Фишера $-F_{\mathrm{T}}$ :

$$
F=\frac{S_{a d}^{2}}{S^{2}(y)}\left\langle F_{T}\left(P ; f_{1} ; f_{2}\right) .\right.
$$

Для этого:

- рассчитывался выход $\hat{y}_{u}$ для каждого варианта опыта по уравнению, из которого исключены незначимые члены;

- находилась разность $\left|\hat{y}_{u}-\bar{y}_{u}\right|$;

- рассчитывалась дисперсия неадекватности по формуле:

$$
S_{a d}^{2}=\frac{\sum_{u=1}^{N}\left|\hat{y}_{u}-\bar{y}_{u}\right|^{2}}{N-N^{\prime}},
$$

где $N^{\prime}$ - число значимых коэффициентов в уравнении регрессии. Рассчитывалось $F$ - отношение по формуле:

$$
F=\frac{S_{a d}^{2}}{S^{2}(\bar{y})} .
$$

Сравнивалось полученное значение F-отношения с табличным значением $F_{\text {т }}\left(P ; f_{1}\right.$; $f_{2}$ ) критерия Фишера при степенях свободы $f_{1}=N(m-1)$ и $f_{2}=N-N^{\prime}$.

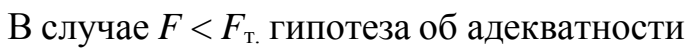
представления результатов эксперимента линейным уравнением регрессии принималась.

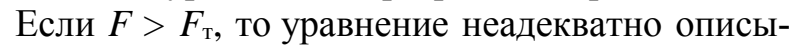
вало экспериментальные данные, точность описания процесса данным уравнением значительно ниже той точности, с которой получены экспериментальные результаты.

На практике часто оказывается, что линейное уравнение регрессии адекватно описывает опытные данные, поставленные в точках 
плана, но неудовлетворительно характеризует внутреннюю область изучаемого факторного пространства. Если дополнительно к плану поставить в нескольких повторностях $\left(m_{0}\right)$ опыт в центре эксперимента, то, не приступая даже к расчету всех (кроме $b_{0}$ ) оценок коэффициентов уравнения, можно судить о возможности описания процесса уравнением без включения в него квадратичных членов.

Оценкой суммарного квадратичного эффекта факторов являлась разность:

$$
\left|\bar{y}_{0}-b_{0}\right|=\sum_{k=1}^{n} b_{i i} .
$$

Если эта разность при заданной вероятности значима, то осуществляется переход к планам второго порядка, позволяющим получить квадратичное уравнение процесса. Значимость разности $\left(\bar{y}_{0}-b_{0}\right)$ устанавливалась при помощи определения доверительной ошибки этой разности: $\varepsilon\left(y_{0}-b_{0}\right)=t(P ; f) S\left(\bar{y}_{0}-b_{0}\right) \quad(17) \quad$ и сравнивалась она с величиной этой разности.

Процедура такой проверки основывалась на дисперсионном анализе, осуществляемом в следующем порядке:

Рассчитывалась среднеарифметическая оценка результата опыта в центре эксперимента:

$$
\bar{y}_{u}=\frac{1}{m} \sum_{k=1}^{m_{0}} y_{k u},
$$

Рассчитываем оценку дисперсии:

$$
S^{2}\left(y_{0 k}\right)=\frac{\sum_{k=1}^{m}\left(y_{k 0}-\bar{y}_{u}\right)^{2}}{\left(m_{0}-1\right)} .
$$

Находилась оценка дисперсии среднего результата, полученного в центре плана по формуле:

$$
S^{2}\left(\bar{y}_{u}\right)=\frac{S^{2}\left(y_{0 k}\right)}{m_{0}} .
$$
ента $b_{0}$ :

Находилась оценка дисперсии коэффици-

$$
S^{2}\left(b_{0}\right)=\frac{S^{2}(\bar{y})}{N}=\frac{S^{2}\left(y_{k}\right)}{m N} .
$$

B соответствии с законом накопления ошибок записывалось:

$$
S^{2}\left(\bar{y}_{u}-b_{0}\right)=S^{2}\left(\bar{y}_{u}\right)+S^{2}\left(b_{0}\right)=\frac{S^{2}\left(y_{0 k}\right)}{m_{0}}+\frac{S^{2}\left(y_{k}\right)}{N m} .(
$$

Если оценки $S^{2}\left(y_{0 k}\right)$ и $S^{2}\left(y_{k}\right)$ однородны, то средневзвешенная оценка дисперсии единичного результата определялась по формуле:

$$
S^{2}=\frac{S^{2}\left(y_{0 k}\right)\left(m_{0}-1\right)+S^{2}\left(y_{k}\right) N(m-1)}{\left(m_{0}-1\right)+N(m-1)}
$$

которая может быть получена с числом степеней свободы $f=\left(m_{0}-1\right)+N(m-1)$.

На завершающем этапе рассчитываем по формуле:

$$
\begin{gathered}
S^{2}\left(\bar{y}_{u}-b_{0}\right)=S^{2}\left(\frac{1}{m_{0}}+\frac{1}{N m}\right)= \\
=S^{2} \frac{N m+m_{0}}{N m m_{0}}
\end{gathered} .
$$

Доверительная ошибка разности $\left(\bar{y}_{0}-b_{0}\right)$ равна:

$$
\varepsilon\left(\bar{y}_{u}-b_{0}\right)=t(P ; f) S\left(\bar{y}_{u}-b_{0}\right) .
$$

Если $\left.\varepsilon\left(\bar{y}-b_{0}\right)>\mid \bar{y}_{u}-b_{0}\right) \mid$, то квадратичные эффекты в уравнении не представлялись. Лишь в случае, если $N>N^{\prime}$, представляется возможным судить об адекватности уравнения, рассчитывая F-отношение.

Если $\left.\varepsilon\left(\bar{y}-b_{0}\right)<\mid \bar{y}_{u}-b_{0}\right) \mid, \quad$ то с заданной вероятностью $P$ считаем эту разность значимой, следовательно невозможно по результатам реализации плана ПФЭ $2^{\mathrm{R}}$ получить хорошую математическую модель процесса. Проведенные эксперименты показали, что данное неравенство выполняется, поэтому необходимо вывести уравнение регрессии второго порядка.

Используя метод наименьших квадратов и закон накопления ошибок, дисперсия коэффициентов регрессии рассчитывается по зависимости:

$$
S^{2}\left(b_{i}\right)=\frac{\left(\sum_{u=1}^{N} x_{i u}^{2}\right)^{\prime}}{\Delta} S^{2}(y),
$$

где $\left(\sum_{u}^{N} x_{i u}^{2}\right)^{\prime}-$ алгебраическое дополнение диагонального элемента $\sum_{u=1}^{N} x_{i u}^{2}$ в определителе системы нормальных уравнений $\Delta$; $S^{2}(y)$ - оценка дисперсии воспроизводи-мости результатов опыта. 
Так как план эксперимента осуществляется в т повторностях, то в формулу (7) войдет средняя оценка дисперсии среднего результата:

$$
S^{2}(\bar{y})=\frac{\sum_{u=1}^{N} \sum_{k=1}^{m}\left(y_{u k}-\bar{y}_{u}\right)^{2}}{N(m-1)}
$$

с числом степеней свободы $f=N(m-1)$. Тогда:

$$
\begin{aligned}
& S^{2}\left(b_{0}\right)=a_{0} S^{2}(y), \\
& S^{2}\left(b_{i}\right)=a_{1} S^{2}(y), \\
& S^{2}\left(b_{i j}\right)=a_{2} S^{2}(y), \\
& S^{2}\left(b_{i i}\right)=a_{3} S^{2}(y),
\end{aligned}
$$

Окончательно для ПФЭ $2^{3}$ :

$$
\begin{aligned}
& S^{2}\left(b_{i}\right)=0,07322 S^{2}(y), \\
& S^{2}\left(b_{i j}\right)=0,125 S^{2}(y), \quad . \\
& S^{2}\left(b_{i i}\right)=0,0597 S^{2}(y)
\end{aligned}
$$

\section{ЛИТЕРАТУРА}

1 Герасимов Д.А., Кравец О.Я., Олейникова С.А. Разработка системы сбора статистики для среды имитационного моделирования // Вестник Воронежского государственного технического университета. 2010. Т.6. № 1. С. 88-91.

2 Свистула А.Е. Двигатели внутреннего сгорания: учебное пособие. Барнаул: Изд-во АлтГТУ, 2009. $81 \mathrm{c.}$

3 Сирота А.А. Компьютерное моделирование сложных систем. Воронеж: Изд-во Воронеж. гос. ун-та, 2006. 248 с.

4 Туревский И.С. Техническое обслуживание и ремонт автомобильного транспорта. Введение в специальность: учебное пособие для студентов учреждений среднего профессионального образования. М.: ФОРУМ : ИНФРАM, 2006. 192 c.

5 Чайнов Н.Д,. Иващенко Н.А., Краснокутский А.Н., Мягков Л.Л. Конструирование двигателей внутреннего сгорания: Учебник для студентов высших учебных заведений, обучающихся по специальности "Двигатели внутреннего сгорания" направления подготовки "Энергомашиностроение". М.: Машиностроение, 2008. $496 \mathrm{c}$.
Если какой-либо из линейных коэффициентов или оценок межфакторного взаимодействия оказывается незначимым, то такой коэффициент (или коэффициенты) исключается из уравнения без пересчета остальных коэффициентов (линейные коэффициенты и оценки эффектов межфакторных взаимодействий определялись независимым образом). Исключение из уравнения незначимого квадратичного коэффициента вызывает необходимость пересчета свободного члена уравнения и других квадратичных эффектов.

\section{Заключение}

Спланирован полный факторный эксперимент ПФЭ позволяет определять взаимодействие двух факторов, которое и проявляется при одновременном варьировании этих факторов при действие каждого из них на выход в зависимости от уровня их нахождения.

\section{REFERENCES}

1 Gerasimov D.A., Kravets O. Ya., Oleinikova S.A. Development of statistical collection system for the simulation environment. Vestnik Voronezhskogo gosudarstvennogo tekhnicheskogo universiteta [Bulletin of Voronezh State Technical University] 2010, vol. 6, no. 1, pp. 88-91. (in Russian).

2 Svistula A.E. Dvigateli vnutrennego sgoraniya [Internal combustion engines] Barnaul, AltGTU, 2009. 81 p. (in Russian).

3 Sirota A.A. Komp'yuternoe modelirovanie slozhnykh sistem [Computer simulation of complex systems] Voronezh, Izd-vo Voronezh. gos. un-ta, 2006. 248 p. (in Russian).

4 Turevskii I.S. Tekhnicheskoe obsluzhivanie i remont avtomobil'nogo transporta. Vvedenie v spetsial'nost' [Maintenance and repair of the motor transport. Introduction to specialty: manual for students of establishments of secondary professional education] Moscow, FORUM, INFRA-M, 2006. 192 p. (in Russian).

5 Chainov N.D., Ivashchenko N.A., Krasnokutsky A.N., Myagkov L.L. Konstruirovanie dvigatelei vnutrennego sgoraniya [Designing of internal combustion engine: textbook for students of the higher educational institutions trained on a speciality "Internal combustion engines" of a direction of preparation "Power-mechanical engineering"] Moscow, Mashionostroenie, 2008. 496 p. (in Russian). 


\section{СВЕДЕНИЯ ОБ АВТОРАХ}

Евгений Д. Чертов д. т. н., профессор, кафедра технической механики, Воронежский государственный университет инженерных технологий, пр-т Революции, 19, г. Воронеж, 394036, Россия

Андрей А. Луканин к. т. н, доцент, военно-воздушная академия имени профессора Н. Е. Жуковского и Ю. А. Гагарина, ул. Старых Большевиков, 54А, Воронеж, 394064, Россия.

Алексей И. Овчинников младший научный сотрудник, военно-воздушная академия имени профессора Н. Е. Жуковского и Ю. А. Гагарина, ул. Старых Большевиков, 54A, Воронеж, 394064, Россия, miliman @inbox.ru

Игорь А. Авцинов д. т. н., профессор, кафедра информационных и управляющих систем, Воронежский государственный университет инженерных технологий, пр-т Революции, 19, г. Воронеж, 394036, Россия

\section{КРИТЕРИЙ АВТОРСТВА}

Евгений Д. Чертов консультация в ходе исследования Андрей А. Луканин консультация в ходе исследования Алексей И. Овчинников обзор литературных источников по исследуемой проблеме, провёл эксперимент, выполнил расчёты

Игорь А. Авцинов консультация в ходе исследования

КОНФЛИКТ ИНТЕРЕСОВ

Авторы заявляют об отсутствии конфликта интересов.

ПОСТУПИЛА 25.07.2016

ПРИНЯТА В ПЕЧАТЬ 18.08.2016

\section{INFORMATION ABOUT AUTHORS}

Evgenii D. Chertov doctor of technical sciences, professor, technical mechanics department, Voronezh state university of engineering technology, Revolution Av., 19, Voronezh, 394036, Russia

Andrei A. Lukanin candidate of technical sciences, associate Professor, air force Academy named after Professor N. E. Zhukovsky and Y. A. Gagarin, street of Old Bolsheviks, building 54A, Voronezh, 394064, Russia.

Aleksei I. Ovchinnikov research associate, air force Academy named after Professor N. E. Zhukovsky and Y. A. Gagarin, street of Old Bolsheviks, building 54A, Voronezh, 394064, Russia, miliman @inbox.ru

Igor' A. Avtsinov doctor of technical sciences, professor, information and control systems department, Voronezh state university of engineering technology, Revolution Av., 19, Voronezh, 394036, Russia

\section{CONTRIBUTION}

Evgenii D. Chertov consultation during the study Andrei A. Lukanin consultation during the study Aleksei I. Ovchinnikov review of the literature on an investigated problem, conducted an experiment, performed computations

Igor' A. Avtsinov consultation during the study

\section{CONFLICT OF INTEREST}

The authors declare no conflict of interest.

RECEIVED 7.25.2016

ACCEPTED 8.18.2016 